\title{
'Brand Placement' en los videoclips del Billboard Hot 100: ¿integración o imposición de marcas?
}

\section{Brand Placement in Billboard Hot 100 Music Videos: Brand Integration or Imposition?}

\author{
Cande Sánchez Olmos \\ Jesús Segarra Saavedra \\ Tatiana Hidalgo Marí \\ Universidad de Alicante (España)
}

Este artículo analiza el brand placement del top 30 de la lista de éxitos Billboard Hot 100 de 2016. Para ello se proponen los siguientes objetivos específicos: conocer qué artistas, marcas y productos aparecen en los hits internacionales; observar el tiempo que ocupan las marcas en los videoclips y analizar si las marcas se integran o se imponen forzando la narrativa del videoclip. La metodología —que combina técnicas cualitativas y técnicas cuantitativasse inicia con un análisis de contenido que pretende medir la frecuencia y el modo en que las marcas son integradas en los videoclips. En segundo lugar, se aplica un análisis cualitativo con perspectiva semiótica para observar la integración de las marcas en el discurso audiovisual. La muestra se centra en la lista de éxitos Billboard, la más importante de la industria de la música a escala internacional en 2016, un año que destaca por la recuperación del sec-
This paper analyses brand placement in the top 30 music videos on the Billboard Hot 100 chart in 2016. To that end, three specific objectives are proposed. First, the paper will identify the artists, brands and products that appear in these international hits. Second, the paper will focus on the duration of time occupied by the brands in the music videos. Finally, the article will examine whether the brands have been integrated into or, on the contrary, imposed on and forced into the narrative of the music video. The methodology, which combines qualitative and quantitative techniques, begins with a content analysis intended to measure the frequency and way in which the brands are integrated into the music videos. Then a qualitative analysis will be carried out from a semiotic perspective in order to discern the degree of integration or imposition of brands in the audiovisual discourse of the music video. The sample is drawn 
tor discográfico. Los resultados apuntan que el videoclip se convierte en un soporte promocional de especial interés para las marcas y permiten confirmar que la presencia de las marcas varía desde un emplazamiento impuesto a una integración sutil, pero perfectamente reconocibles por la audiencia.

Palabras clave: emplazamiento publicitario, música y publicidad, emplazamiento del producto, publicidad en YouTube, videoclip. from the Billboard Hot 100 chart, which was the most important list in the international music industry in 2016, a year that stands out because of the recovery of the record industry. The results indicate that music videos have become an advertising format of special interest to brands and confirm that the presence of brands ranges from imposed placement to subtle integration, but that they are perfectly recognisable to the audience in either case.

Keywords: advertising placement, music and advertising, product placement, advertising on YouTube, music video.

$\Lambda$ nte el rechazo a una publicidad tradicional que interrumpe el entretenimiento, los publicistas buscan vías de conexión con los consumidores menos invasivas a través del brand placement, técnica publicitaria que consiste en la integración de una marca o servicio en un contenido audiovisual de entretenimiento (Pino y Olivares, 2006; Lehu, 2007). A través del mismo, las marcas se integran en la narrativa y escenarios de una película, programa de televisión o videoclip con el objetivo de generar significados y asociaciones positivas que inviten al usuario a comprar la marca, o al menos a tenerla presente en su proceso de decisión de compra.

Teniendo en cuenta la importancia que tiene la música para las personas y observando el creciente interés de las marcas por integrar sus productos en la música, esta investigación tiene como objetivo principal analizar la presencia de marcas en los videoclips del Billboard Hot 100 en 2016. Existen antecedentes que invitan a una investigación más profunda sobre el emplazamiento de productos en vídeos musicales. Los datos registrados en 2015 indicaron que de los 378 videoclips de esta lista el 81\% mostró al menos el emplazamiento de una marca, lo que supone un crecimiento del $25 \%$ respecto al 2014. En total fueron identificadas 650 marcas diferentes en esos 378 vídeos que alcanzaron cerca de los 40 mil millones de visionados (Sánchez, 2016).

Los videoclips son una de las manifestaciones estéticas más nutritivas de la cultura popular. De hecho, de los 100 videoclips más vistos en la historia de YouTube, 90 se corresponden con videoclips oficiales de artistas y bandas reconocidas a escala internacional; el resto son videos infantiles, varios de los cuales incluyen música (MyTop100videos, 2018). Esto explica por qué los videoclips se 
han convertido en uno de los contenidos de entretenimiento más preciados por las marcas para integrar sus productos.

Tanto la industria discográfica como la publicitaria han sufrido cambios importantes desde el desarrollo de internet. Del lado de la música, el sector ha sufrido una crisis debido al cambio en los modos en los que se produce, distribuye y consume la música. Tanto es así que hasta 2012 no se registró el primer valor positivo en el volumen de negocio de la industria musical desde 1999. Es más, desde finales de siglo hasta 2016, la industria discográfica ha sufrido una caída del 40\% de los ingresos. En 2015 se confirmó que los acuerdos con marcas han sido una de las áreas de inversión de mayor crecimiento para el sector. De hecho, los ingresos por sincronizaciones publicitarias y audiovisuales suponen actualmente el 2\% de los ingresos, que alcanzaron en 2016 los 15,700 millones de dólares (IFPI, 2016). Las discográficas han establecido departamentos especializados para llegar a acuerdos de brand placement con marcas. Aun así, estas cifras están lejos de alcanzar los más de 20 millones de dólares que ingresó la industria de la música en 2005, lo que implica que las marcas son para la música una alternativa para mejorar sus ingresos.

Del lado de la publicidad, las marcas se han encontrado que las nuevas tecnologías han fragmentado las audiencias y han posibilitado que los espectadores cuenten con numerosas herramientas para huir de la publicidad invasiva (Pino, Castelló y Ramos, 2013). Las audiencias se han vuelto escépticas a los formatos publicitarios tradicionales que están basados en la repetición y la interrupción (Regueira, 2012). El rechazo a la publicidad ha sido uno de los grandes problemas de los publicistas y es también uno de los temas que más literatura ha generado. El desarrollo de internet, el surgimiento de nuevas cadenas de televisión y la TDT (Televisión Digital Terrestre) provocaron una fragmentación de las audiencias. La tecnología posibilita huir más fácilmente de la publicidad (Baek y Morimoto, 2012; Holden, 2007). De entre todos los medios, la televisión es el medio más afectado por este rechazo (Dix y Phau, 2010).

En plena evolución de la industria publicitaria hacia la postpublicidad (Solana, 2010), el marketing online — que camina entre las disciplinas de la comunicación persuasiva y la informática - tiende a centrar sus esfuerzos en el posicionamiento en buscadores a través de, entre otros, el videomarketing y el branded content. Por ello, canales como YouTube adquieren gran relevancia en la estrategia de medios de los anunciantes. De hecho, la emancipación del videoclip de la televisión debido al desarrollo de plataformas de video como YouTube y Vimeo ha motivado el crecimiento del brand placement.

Por último, es importante justificar la muestra de esta investigación. El Hot 100 del Billboard es una lista que posiciona del 1 al 100 las canciones de mayor éxito de ventas en la industria discográfica en EE UU desde 1955, por lo que está considerada como la biblia de la industria de la música. La influencia de esta lista se ha mantenido intacta y sigue siendo la lista de referencia para la música a la hora de lanzar nuevos artistas. Por esta razón, las marcas se ven atraídas por los altos índices de popularidad y de éxito de las bandas que forman parte de ella, ya que son garantía de visibilidad y de influencia. Las variables de análisis de esta investigación presentan una metodología sólida y exhaustiva para analizar qué 
66 artistas, marcas, productos, tiempo de presencia de las marcas en pantallas y tipo de emplazamiento. Por ello, consideramos conveniente comenzar por la lista de mayor éxito que existe en el sector de la industria de la música para establecer las bases de una investigación que en un futuro pretende ser más ambiciosa analizando la evolución de esta técnica publicitaria en la primera década de YouTube. Por otro lado, esta lista de éxito del Hot 100 del Billboard coincide con la lista de videoclips más visionados en YouTube, lo que justifica la investigación de los videos que consiguen mayor atracción de la audiencia.

En definitiva, si el brand placement es una técnica publicitaria que está creciendo de forma constante y la industria de la música ha encontrado una alternativa en las marcas para mejorar sus ventas, es necesario analizar cómo se integran las marcas en los videoclips. Si la música emociona, comunica y crea identidad, es necesario averiguar hasta qué punto las marcas influyen en la creación de estos significados.

\section{MARCO TEÓRICO}

\section{EL VIDEOCLIP EN LA ACTUALIDAD}

Viñuela (2013) entiende el videoclip como un texto audiovisual de compleja definición dentro de los fenómenos que conforman la industria musical, si bien es cierto que tradicionalmente se ha considerado al videoclip como un producto de una discográfica cuyo objetivo es vender la canción a través de la grabación de la música con unas imágenes (Goodwin, 1993). Vernallis (2013) cree que esta definición podría ser válida para las primeras décadas del desarrollo del videoclip, que surgió con el nacimiento de la MTV en 1981, pero considera que ya no cubre la diversidad de formatos que ha surgido en la segunda oleada estética de YouTube. La autora define el videoclip como el producto audiovisual que la audiencia reconoce como tal. Añade que existe una constelación de elementos ocultos que están pidiendo ser descifrados con la finalidad de dar sentido a los nuevos videoclips. La producción de videoclips por parte de las marcas podría ser uno de esos elementos ocultos, una nueva categoría de vídeos musicales.

La línea que separa a la publicidad audiovisual del videoclip se difumina puesto que los vídeos musicales funcionan como soportes en los que las marcas integran sus productos desde su desarrollo en MTV. Así las cosas, además de promocionar la canción, persuaden y seducen a la audiencia para que desee toda la parafernalia que se muestra en el videoclip: ropa, complementos, etc. (Kaplan, 1987; Englis, 1991; Goodwin, 1993). Esta tendencia se ha mantenido en la era YouTube.

\section{EL 'BRAND PLACEMENT' EN EL VIDEOCLIP}

Nos referimos al brand placement como la integración de una marca o un servicio en un contenido audiovisual (Pino y Olivares, 2006; Lehu, 2007). Académicos y profesionales coinciden en destacar que las marcas encuentran cada vez más 
dificultad para ser escuchadas porque los consumidores están saturados debido al bombardeo de mensajes informativos y publicitarios (Madinabeitia, 2005). Tanto es así que los ingresos por brand placement superaron por vez primera en 2014 los 10.000 millones de dólares en el mercado americano, según la consultora PQ Media (2018). El mismo informe indica que desde 2009, año en el que se registró la mayor caída por ingresos publicitarios como consecuencia de la crisis, el brand placement ha ido creciendo hasta superar en 2016 los 14.000 millones de dólares. Por tanto, para PQ Media el brand placement es la estrategia de marketing que ha ofrecido una mayor estabilidad en el último siglo porque si está bien ejecutada consigue atraer a la audiencia y establecer conexiones con unos potenciales consumidores que no perciben el contenido de entretenimiento como publicidad tradicional. Es más, de todos los contenidos en los que se integran las marcas (películas, programas de televisión y videoclips), la integración de marcas en los videos musicales, que alcanzó aproximadamente los 1.000 millones de dólares en 2016, ha sido el sector de mayor crecimiento, según PQ Media. En este contexto no extraña que Marques y Sedeño (2017) hablen de ubicuidad de marcas en los videoclips.

Pero esta técnica no está exenta de polémica dado que la audiencia, en principio, no es consciente del consumo de este tipo de publicidad, a no ser que aparezca la sobreimpresión "Emplazamiento publicitario", como debe ocurrir en televisión. En relación con el brand placement, Hudson y Hudson (2006) plantearon preguntas de investigación relacionadas con la ética y la presencia de marcas en el entretenimiento, ya que el emplazamiento de productos escapa a la regulación publicitaria convencional más allá de la televisión. ${ }^{1}$ Sin embargo, el brand placement en los videoclips aparece analizado de forma tangencial en comparación con la dedicación que reciben sectores como el cine y los programas de televisión. Por ello, es necesario profundizar en la presencia de marcas en los videoclips.

En varias investigaciones Thornton y Burkhalter $(2011,2015)$ demostraron que el brand placement supone una gran oportunidad para las marcas porque despierta el interés de las personas de los productos integrados en un videoclip. En este sentido, destacan las investigaciones de Krishen y Sirgy (2016), Matthes y Naderer (2016), Smit, Reijmersdal y Neijens (2009) y Regueira (2012), las cuales indagan en los efectos que producen el brand placement en los usuarios y la integración de marca en el entretenimiento. Los resultados indican que el brand

1 El emplazamiento en televisión sí está regulado en el artículo 17 de la Ley General de la Comunicación Audiovisual de 2010 que se puede consultar en <https://goo.gl/4jYHxY>. Por tanto, el emplazamiento de marcas en videoclips debería aparecer acompañado de la sobreimpresión "Emplazamiento publicitario" tal y como ocurre en programas de televisión, cosa que no ocurre en YouTube. Esto muestra un desequilibrio entre diferentes medios. Pero, además, se da la circunstancia de que el videoclip, al no ser un formato publicitario, sino producido por la industria musical para promocionar bandas y solistas, no ha estado acompañado de dicha sobreimpresión que indica que es un mensaje publicitario ni en televisión ni en YouTube. 
68 placement genera asociaciones positivas en las mentes de los consumidores. Sin embargo, estas relaciones no implican una intención de compra, y, por lo tanto, se cuestiona su efectividad (Regueira, 2012).

El brand placement gana terreno porque la imagen de la publicidad se ha deteriorado; en los últimos años el hastío que ha ido produciendo ha crecido de manera considerable (Moor, 2003; Regueira, 2012). Incluso existen consumidores que se han organizado en comunidades en las que comparten la aversión por las marcas y su dominación corporativista. Los consumidores luchan contra las marcas que explotan, no son éticas, destructivas o monopolizan los mercados (Klein, 2000). Internet ha posibilitado que estas comunidades hayan crecido y aumentado, han conseguido mayor coalición, unión espacial y han aprovechado las herramientas de la red para expandir el activismo (Hollenbeck y Zinkhan, 2006).

Desde una perspectiva crítica algunos investigadores han detectado que el emplazamiento de producto en el videoclip es demasiando obvio, lo que resta credibilidad y valor estético al discurso audiovisual y muestra una dependencia del video musical de los objetivos comerciales de las marcas (Pérez, Navarrete y Gómez, 2014).

En la misma línea crítica, otras investigaciones (Soba y Aydin, 2013) denuncian cuestiones poco éticas que plantea esta técnica de marketing. Existen diferentes factores que condicionan el éxito de la integración de una marca en un contenido musical: la calidad y creatividad, la familiaridad que el usuario tenga con la marca, el grado de afinidad que los espectadores tengan con el artista, la sutileza con la que haya sido incluida la marca... Por otro lado, existe un debate en torno a la ética de esta estrategia comercial porque el brand placement es un tipo de publicidad que no se presenta como tal, ni aparece en los controles de inversión publicitaria como los de InfoAdex. Además, obliga a las audiencias a una exposición, en ocasiones, desmesurada de marcas en contenidos de entretenimiento (Soba y Aydin, 2013).

Cabe destacar las investigaciones que recientemente se han realizado sobre el brand placement en videoclips producidos por las propias marcas, es decir, branded content musical. En este caso, las marcas ajenas a la industria musical producen sus propios videoclips para tener un mayor control del emplazamiento de sus productos. Por tanto, se trata de videoclips publicitarios en los que es imposible diferenciar la línea que separa la música de la publicidad. Dicho en otras palabras, estos contenidos son más que música, son musicidad, es decir, un contenido en el que coexisten la música y la publicidad. En cuanto a sectores, la moda es el más activo en la producción de branded content musical (Sánchez, 2018).

En definitiva, si bien las investigaciones sobre brand placement profundizan en esta técnica desde diferentes perspectivas —efectividad, recuerdo, efectos en la audiencia, sus luces y sombras-, apenas existen estudios que analicen formalmente cómo se produce el emplazamiento de productos. En este sentido, el presente artículo tiene como objetivo completar la escasez de investigaciones sobre brand placement en videoclips. 


\section{DISEÑO DE LA INVESTIGACIÓN Y METODOLOGÍA}

El objetivo general de la investigación es conocer cómo se integran las marcas en el discurso audiovisual de los videoclips. La muestra de la investigación está compuesta por 30 videoclips (tabla 1), ${ }^{2}$ que ocupan los primeros puestos de la lista del Hot 100 del Billboard del 2016.

Tabla 1. Muestra de la investigación

\begin{tabular}{|c|c|}
\hline Videoclip & Artista \\
\hline 1. "Love Yourself (Purpose: The Movement)" & Justin Bieber \\
\hline 2. "Sorry" & Justin Bieber \\
\hline 3. "One Dance" & Drake ft. Wizkid and Kyla \\
\hline 4. "Work" & Rihanna ft. Drake \\
\hline 5. "Stressed Out" & Twenty One Pilots \\
\hline 6. "Panda" & Desiigner \\
\hline 7. "Hello" & Adele \\
\hline 8. "Don't Let Me Down" & The Chainsmokers ft. Daya \\
\hline 9. "Can't Stop the Feeling!" & Justin Timberlake \\
\hline 10. "Closer" & The Chainsmokers ft. Halsey \\
\hline 11. "Cheap Thrills" & Sia ft. Sean Paul \\
\hline 12. "7 Years" & Lukas Graham \\
\hline 13. "Needed Me" & Rihanna \\
\hline 14. "My House" & Flo Rida \\
\hline 15. "I Took a Pill in Ibiza" & Mike Posner \\
\hline 16. "Work from Home" & Fifth Harmony ft. Ty Dolla \$ign \\
\hline 17. "This Is What You Came For" & Calvin Harris ft. Rihanna \\
\hline 18. "Cake by the Ocean" & DNCE \\
\hline 19. "Me, Myself \& I" & G-Eazy x Bebe Re \\
\hline 20. "Ride" & Twenty One Pilots \\
\hline 21. "Heathens" & Twenty One Pilots \\
\hline 22. "Pillowtalk" & Zayn \\
\hline
\end{tabular}

2 Lista de reproducción con videoclips analizados disponible en <https://bit. ly/2zmN3Zx>. 


\begin{tabular}{|c|c|c|}
\hline 70 & Videoclip & Artista \\
\hline \multirow{8}{*}{ 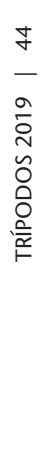 } & 23. "Stitches" & Shawn Mendes \\
\hline & 24. "Hotline Bling" & Drake \\
\hline & 25. "Cold Water" & Major Lazer ft. Justin Bieber and M $\varnothing$ \\
\hline & 26. "Send My Love (To Your NewLover)" & Adele \\
\hline & 27. "Roses" & The Chainsmokers ft. Rozes \\
\hline & 28. "Treat You Better" & Shawn Mendes \\
\hline & 29. "Too Good" & Drake ft. Rihanna \\
\hline & 30. "Low Life" & Future ft. The Weeknd \\
\hline
\end{tabular}

Fuente: elaboración propia a partir del Billboard Hot 100.

Para ello planteamos cuatro objetivos específicos:

1. Conocer qué artistas, marcas y productos aparecen en los hits internacionales de la lista de éxito más importante de la industria de la música a escala mundial en 2016.

2. Medir durante cuánto tiempo están presentes las marcas en los videoclips.

3. Observar la visibilidad del logo en el producto integrado con la finalidad de analizar la integración o imposición de la marca.

4. Analizar cómo se integran las marcas en los videoclips con la finalidad de observar si las marcas son integradas o impuestas en los videoclips. Para ello establecemos cinco categorías (tabla 2) que indican de forma descendente la prominencia de la marca en la pantalla:

Tabla 2. Categorías formales de integración de marcas

\begin{tabular}{l|l|l} 
Categoría & Category & Descripción \\
\hline Primerísimo primer plano & Close up & $\begin{array}{l}\text { Emplazamiento manifiesto de la marca, más impuesto que } \\
\text { integrado. }\end{array}$ \\
\hline Evidente & Obvious & $\begin{array}{l}\text { Emplazamiento obvio. Se observa imposición de la marca en } \\
\text { segundo plano. }\end{array}$ \\
\hline Sutil & Subtle & $\begin{array}{l}\text { Emplazamiento sutil e integrado en el videoclip, pero } \\
\text { condicionando la narrativa. }\end{array}$ \\
\hline De fondo & Background & $\begin{array}{l}\text { Emplazamiento de fondo, por ejemplo, en los escenarios. Permite } \\
\text { la identificación de la marca sutilmente en planos largos. }\end{array}$ \\
\hline Discreta & Discret & $\begin{array}{l}\text { Presencia de marcas de una forma discreta, apenas perceptible } \\
\text { por la audiencia. }\end{array}$ \\
\hline & \multicolumn{2}{|l}{$\begin{array}{l}\text { Fuente: elaboración propia. }\end{array}$}
\end{tabular}

La metodología de este proyecto de investigación combina técnicas cualitativas y técnicas cuantitativas. En el caso de las técnicas cualitativas empleamos un análisis de contenido con el objetivo de medir de forma estadística la frecuencia y el 
modo en el que las marcas son integradas en los videoclips a lo largo del periodo establecido. Cuando una marca aparece en pantalla se registran todas las variables con sus correspondientes categorías en un cuestionario Excel creado ad hoc. Esta técnica nos permite la recolección de unos datos exhaustivos de cada marca que aparece en los videoclips. Existen productos en los que no ha sido posible identificar la marca. En este caso, no se contabilizan en las variables, ya que el objetivo es analizar el emplazamiento de marcas por encima del emplazamiento de productos. Una vez seleccionados los registros aplicamos un análisis cuantitativo univariable. Así, se resumen las observaciones de una variable de forma independiente y se comparan valores de distintas variables para expresarlas unas en función de otras (Sierra y Bravo, 2005).

\section{RESULTADOS}

\section{LA IMPORTANCIA DE LOS ARTISTAS COMO PRESCRIPTORES DE MARCAS}

Tras analizar los resultados cuantitativos obtenidos, nos encontramos que, entre los 30 videoclips analizados pertenecientes al Hot 100 de Billboard, los artistas más representativos son los que podemos ver en la imagen 1 .

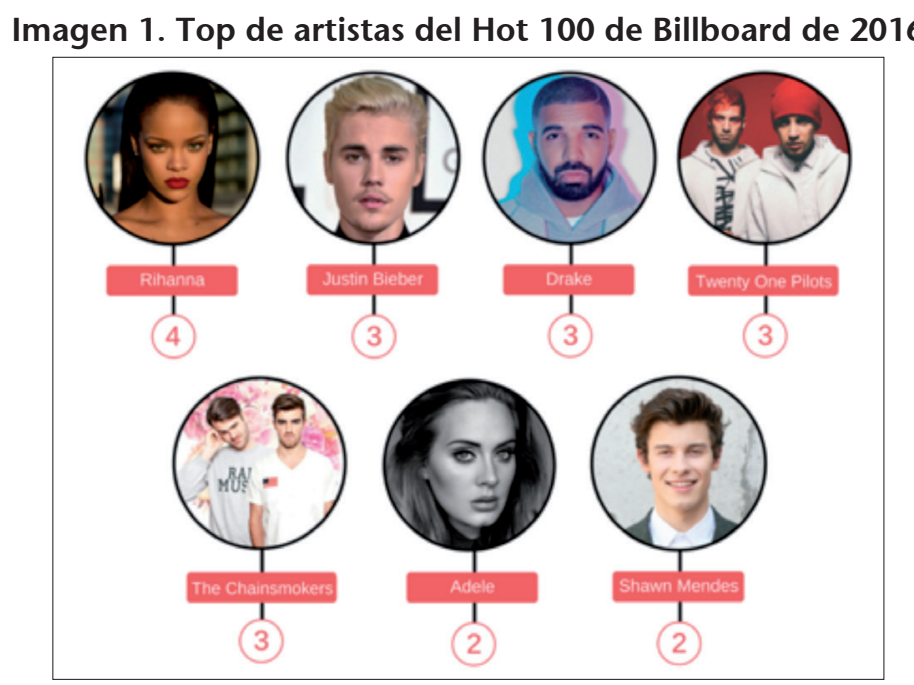

Fuente: elaboración propia a partir del Billboard Hot 100.

Se aprecia que el éxito de un cantante o grupo musical genera, directamente, un interés por parte de la industria publicitaria en sus narraciones, debido al impacto que pueden obtener las inserciones publicitarias en los videoclips de más éxito. Así, observamos que, de los 30 videoclips analizados, 4 de ellos son protagonizados por Rihanna, siendo ella la cantante más relevante en el ranking de videoclips de 2016. Le siguen cantantes como Justin Bieber, Drake, Twenty One Pilots o 
72 The Chainsmokers, que protagonizan tres videoclips respectivamente del total y Adele y Shawn Mendes, que son los protagonistas de 2 videoclips dentro del ranking. Estos resultados demuestran la escasa diversidad musical: solo 7 cantantes representan dos tercios de la lista.

A pesar de que estos son los más representativos por tener más de un videoclip dentro del Hot 100 de Billboard, existen otros cantantes que actúan como prescriptores, precisamente, por el éxito de sus videoclips. Destacables, entre otros, Desiigner ( $6^{\circ}$ en el ranking) Justin Timberlake ( $9^{\circ}$ en el ranking). La tabla 3 recoge el resto de los artistas que adquiere importancia en la muestra y la posición que ocupan en el ranking del Hot 100.

\begin{tabular}{l|l}
\multicolumn{2}{l}{ Tabla 3. Artistas y posición en el 'ranking' } \\
Artista & Número 'ranking' \\
\hline Desiigner & 6 \\
\hline Justin Timberlake & 9 \\
\hline Sia ft Sean Paul & 11 \\
\hline Lukas Graham & 12 \\
\hline Flo Rida & 14 \\
\hline Mike Posner & 15 \\
\hline Fifth Harmony ft Ty Dolla Sign & 16 \\
\hline Calvin Harris ft Rihanna & 17 \\
\hline DNCE & 18 \\
\hline G-Eazy x Bebe Rexha & 19 \\
\hline Zayn Malik & 22 \\
\hline Major Lazer ft Justin Bieber \& MO & 25 \\
\hline Future ft The Weeknd & 30 \\
\hline \multicolumn{2}{|c}{ Fuente: elaboración propia. }
\end{tabular}

\section{MARCAS EN EL VIDEOCLIP: PRESENCIA Y TIEMPOS DE EXPOSICIÓN}

Las marcas más representativas de la muestra se caracterizan por ser marcas conocidas, con importante peso en la industria publicitaria y fácilmente reconocibles por los consumidores. De las marcas registradas en la investigación, nos encontramos con que 8 de ellas tienen una presencia en más de 10 casos, lo que resulta un indicador de la apuesta por el brand placement musical por parte de la marca. Así pues, la marca con mayor número de apariciones es Chevrolet (13 apariciones), seguida de Apple (12 apariciones) y Adidas, Ford y Puma (11 apariciones cada una de ellas). Les siguen BMW, Rolex y Timberland, con 10 apariciones cada una de ellas.

Como se puede apreciar en la tabla 4, a pesar de que solo 8 marcas superan las 10 apariciones en la totalidad de la muestra analizada, muchas otras marcas 
adquieren una presencia notable, si bien es cierto que el número de veces contabilizadas no superan las 10. Hablamos de marcas como Versace (8 apariciones), Samsung (7 apariciones), KTZ, Lamborgini, Nike y Remo (6 apariciones cada una); Converse, Fender, Red Strripe, Shure y Sound Percussion (con 5 apariciones cada una) u otras menos presentes, pero con cierta relevancia en el ranking, como Levi's, Rolls Royce, SJC o Toyota (4 apariciones cada una).

Al margen del sector al que pertenece cada marca, resulta relevante destacar cómo predominan sectores genéricos como la tecnología, el mundo del motor y la moda (deportiva y de lujo, fundamentalmente). En este sentido, el coche es el vehículo que transporta a las personas a la fiesta, es un símbolo de la libertad y del individualismo juvenil, al menos hasta que disponen de casa propia. La música es crucial para atraer a los consumidores porque ofrece la banda sonora de las experiencias vividas en un coche. Por otro lado, el sector de la moda y complementos y el de la música casan especialmente bien por varias razones. Por un lado, ambos imponen unas normas generales a las cuales se pueden adscribir grupos sociales; por otro, ambos dejan sitio a la manifestación del gusto individual. Música y moda son fundamentales para la construcción de la identidad de las personas. Con relación a esto, llama la atención la presencia de marcas competidoras entre sí en el seno de un mismo videoclip, como ocurre en "Sorry", el vídeo musical de Justin Bieber en el que conviven marcas como Adidas, Kappa y Nike, todas vinculadas a la ropa y los complementos deportivos. En este caso, los resultados coinciden con los obtenidos por Sánchez (2018): la moda es uno de los sectores más activos en esta muestra de investigación.

Por la propia naturaleza del videoclip, adquieren importancia marcas pertenecientes al mundo de la música, los instrumentos y los materiales sonoros, que interactúan con la narración de forma armónica y natural, pero sin renunciar al carácter promocional que atribuyen sus apariciones.

Tabla 4. 'Ranking' de marcas y apariciones computadas

\begin{tabular}{l|l} 
Marca & Apariciones \\
\hline Chevrolet & 13 \\
\hline Apple & 12 \\
\hline Adidas & 11 \\
\hline Ford & 11 \\
\hline Puma & 11 \\
\hline BMW & 10 \\
\hline Rolex & 10 \\
\hline Timberland & 10 \\
\hline Versace & 8 \\
\hline Samsung & 7 \\
\hline
\end{tabular}




\begin{tabular}{l|l} 
Marca & Apariciones \\
\hline KTZ & 6 \\
\hline Lamborghini & 6 \\
\hline Nike & 6 \\
\hline Remo & 6 \\
\hline Converse & 5 \\
\hline Fender & 5 \\
\hline Red Stripe & 5 \\
\hline Shure & 5 \\
\hline Sound Percussion & 5 \\
\hline Levi's & 4 \\
\hline Rolls-Royce & 4 \\
\hline SJC Custom Drums & 4 \\
\hline Toyota & 4 \\
\hline
\end{tabular}

En relación con el segundo objetivo, procedemos a analizar el tiempo que ocupa la presencia de las marcas en los videoclips. Para ello, se han registrado los segundos en los que se puede apreciar presencia visual de la marca y se ha relacionado con la totalidad de tiempo visual que ocupa la muestra de análisis. Los resultados apuntan que las marcas están presentes el 53\% del tiempo total, esto son 60 minutos de presencia publicitaria en una totalidad de 114 minutos de videoclips analizados. Los datos confirman, al igual que ocurría en el punto anterior, que existe un interés notable y representativo por el brand placement en los videoclips internacionales y que, de forma progresiva, las marcas están ocupando, cada vez más, el espacio narrativo del videoclip.

Gráfico 1. Presencia de marcas en tiempo

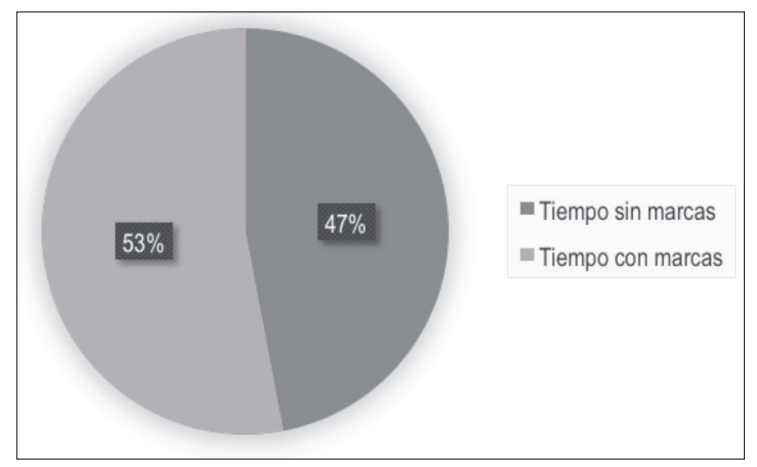

Fuente: elaboración propia. 
Si comentamos la visibilidad de las marcas (gráfico 2), podemos decir que, de la totalidad de veces que aparecen en las piezas audiovisuales, el $81 \%$ de los casos muestran la marca de forma publicitaria, esto es, mostrando de forma clara y sin lugar a duda el logotipo, más bien impuesta que integrada. El 19\% de casos restantes utilizan la marca de forma sugerida, normalmente mediante la promoción de productos evidentes (por ejemplo, el uso de un teléfono Apple, que, a pesar de que no muestra el logo, identifica perfectamente a qué marca pertenece) u otras atribuciones características del producto que, a pesar de no contener el logotipo de forma evidente, hacen una alusión indiscutible a la marca y, por tanto, confirman la presencia publicitaria, aunque de forma sugerida, es decir, integrada en la narrativa.

Gráfico 2. Visibilidad del logotipo de la marca

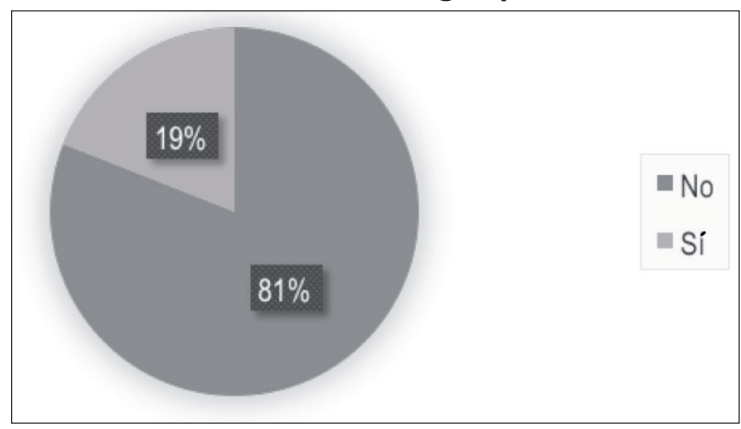

Fuente: elaboración propia.

\section{EMPLAZAMIENTOS PUBLICITARIOS EN EL VIDEOCLIP}

Para dar respuesta al cuarto objetivo, se analizan las formas en que las marcas se integran en la narración del videoclip. Este análisis permite conocer si existe una relación natural entre la marca y la historia o personajes o si, por el contrario, la marca se impone, forzando la narrativa del videoclip.

\section{Gráfico 3. Integración de la marca en la narración}

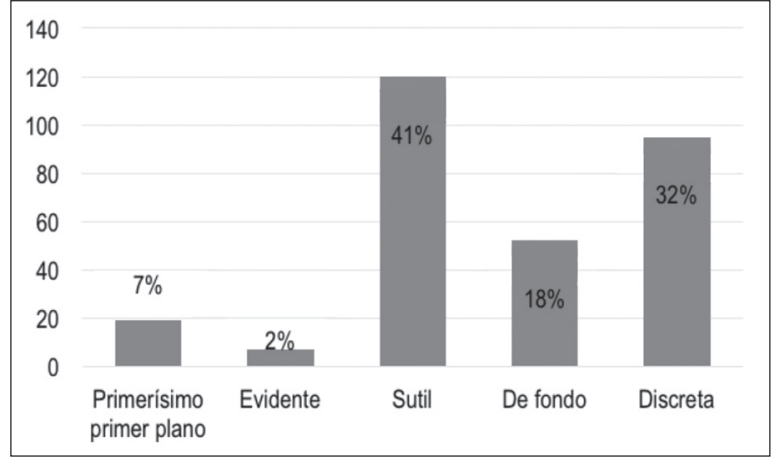

Fuente: elaboración propia. 
76 Según el gráfico 3, la forma en que se emplaza la marca dentro de la narración es variable y dependerá, en gran medida, de las necesidades narrativas y de los objetivos comunicativos publicitarios. En cualquier caso, los datos obtenidos tras el análisis nos permiten afirmar que en el 41\% de los casos (120 apariciones), la marca aparece de forma sutil, sin una necesidad patente de ser protagonista en la narración, sino que, más bien, pretende ser un elemento que interactúa de forma natural con el personaje o con la propia trama. Como ejemplo de esta categoría observamos la camiseta de Gucci del videoclip de Rihanna o las zapatillas de Adidas de Lukas Graham (imagen 2).

Siguiendo en la línea de las apariciones sutiles, el segundo tipo de aparición más relevante es el emplazamiento etiquetado como discreto, que destaca en el 32\% de los casos analizados (95 apariciones). Este tipo de emplazamiento, a pesar de ser muy similar al anterior, se caracteriza por dar protagonismo a la marca como tal, pero de una forma muy neutra, normalmente, mediante el uso homogéneo de la marca o el producto dentro de la narración. En esta categoría destaca la cazadora Levi's 501 blanca que lleva Justin Timberlake o la camiseta Nike Air Jordan que lleva Drake (imagen 2), entre otras prendas de vestir que se observan de forma discreta, pero que no impiden la transmisión de una serie de significados por parte de los músicos a través de dichas marcas.

El emplazamiento de la marca en formato background o en segundo plano es bastante relevante si tenemos en cuenta que se detecta en el 18\% de los casos (52 apariciones). A pesar de no tener el mismo protagonismo que los casos sutiles o discretos, utilizar la marca como decorado, fondo o segundo plano de la narración es algo que se repite continuamente en los videoclips, lo que aporta un posicionamiento muy perceptible a la marca, pero sin alterar la evolución natural de la narración. Podríamos decir que el emplazamiento por background se asemeja a otras formas publicitarias, como el patrocinio o el product placement, ya que la interacción de la marca no es evidente, sino que adquiere un protagonismo secundario, a pesar de que resulta muy visual y evidente. Es el caso de los cereales Cheers que aparecen en las estanterías del supermercado en el videoclip de Justin Timberlake: "Can't Stop This Feeling!". La marca, aunque esté en el background, es completamente reconocible, ya que la disposición de los cereales en un supermercado no coincide con lo que representa este videoclip. Por tanto, la presencia es discreta, pero ha sido creada con el objetivo de que la audiencia la reconozca y se lea familiar a la hora de hacer la compra.

Si bien es cierto que la mayoría de los emplazamientos se caracterizan por su carácter sutil, discreto o en segundo plano (81\% del total de casos analizados), lo cierto es que se detectan nuevas tendencias en la presencia de marcas en los videoclips. Así, podemos encontrar la apuesta por el emplazamiento de cerca o close-up, que se caracteriza por aportar una imagen (normalmente un primer plano) de la marca o el producto. Además, este emplazamiento, que está presente en un 7\% de los casos analizados (19 apariciones en total), resulta muy agresivo puesto que altera la narrativa lineal del videoclip en aras de realizar una exposición o enfoque de la marca de forma directa, una exposición comunicativa que el receptor no puede pasar por alto, dado su carácter evidente. Normalmente, es el propio sujeto de la narración quien actúa como prescriptor, acercando el 
producto a cámara o forzando el plano para que la marca aparezca de cerca en la narración. En este ejemplo encontramos casos como los de la marca de auriculares que pertenece a Apple Beats by Dre y que aparece de manera impuesta más que integrada en el videoclip "Me, Myself \& I", de G-Eazy con Bebe Rexha. Otro caso es la bebida energética Celsius, que no solo prescribe el rapero Flo Rida, sino que además la bebe mientras canta (imagen 2).

Este tipo de emplazamiento se relaciona directamente con el emplazamiento obvio, que está presente solo en un $2 \%$ de los casos (7 apariciones). El emplazamiento obvio sigue la línea agresiva y directa del emplazamiento de cerca o close-up con la diferencia de que no hay un sujeto que se encargue de la presentación de la marca, sino que, más bien, la marca se visualiza en primer plano o plano corto, pero haciéndolo de forma más natural, no tan agresiva, a pesar de que, como el propio nombre de la categoría indica, resulta obvio que hay una intencionalidad en la aparición de la marca y que dicha intencionalidad pretende que la marca sea percibida de cerca, sin dar lugar a dudas sobre su presencia. En esta categoría, destacan las marcas deportivas Everlast en el videoclip de Justin Bieber "Sorry", o las Converse All Star del videoclip de Justin Timberlake "Can't Stop This Feeling!” (imagen 2).

Imagen 2. Ejemplos del tipo de emplazamiento

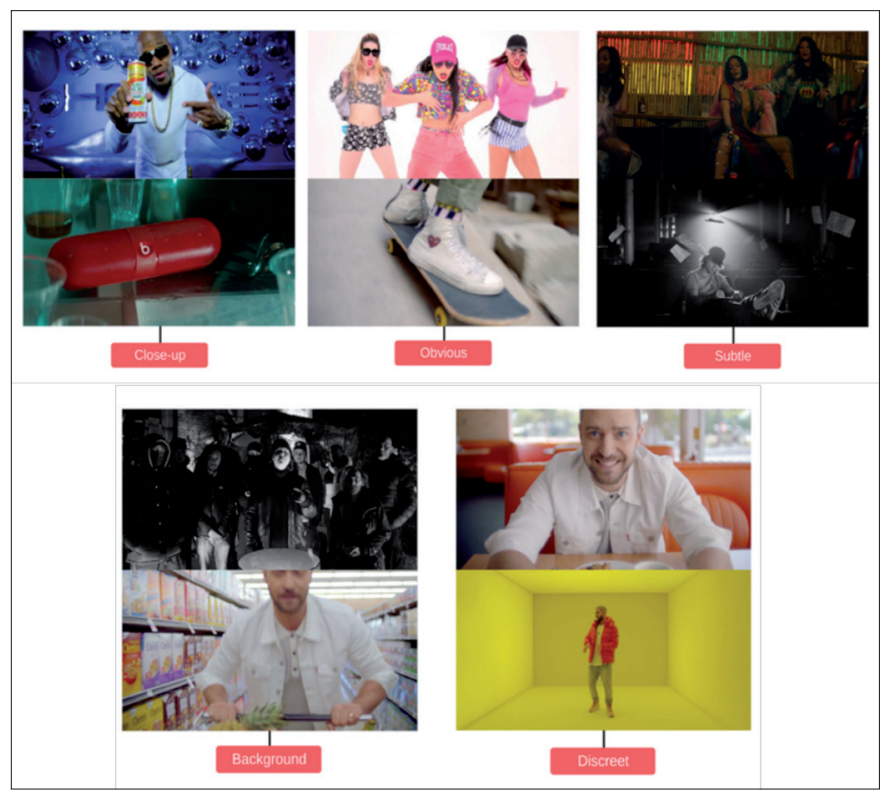

Fuente: elaboración propia a partir de fotogramas de videoclips.

\section{CONCLUSIONES}

Tras la exposición de los datos obtenidos en el análisis podemos afirmar que existe una evidencia en la relación de las marcas con la industria musical y que, 
concretamente, el videoclip se convierte en un soporte narrativo que propicia ese emplazamiento publicitario, tanto por su alcance como por su carácter audiovisual y narrativo, un formato híbrido entre la promoción y la creación.

Se observa cómo la figura del artista como prescriptor adquiere especial importancia por dos motivos: en primer lugar, porque por su carácter prescriptor, todas esas comunidades de fans que lo apoyan son público susceptible de consumir sus videoclips; y, en segundo lugar, por la posibilidad de expansión de la marca. Además, resulta relevante cómo los cantantes de más éxito son, además, los que mayor número de emplazamientos publicitarios reciben, lo que confirma por tanto este detalle.

Se constata que las marcas aparecen en más del 50\% del tiempo total de los videoclips, por lo que no se puede pasar por alto la importancia que están adquiriendo dentro de las narraciones audiovisuales musicales. Los datos confirman que existe un interés notable y representativo por el brand placement en videoclips y que, de forma progresiva, las marcas están ocupando, cada vez más, el espacio narrativo del videoclip.

Mención aparte merecen los emplazamientos y sus tipos. Como hemos podido observar, la tendencia es que la presencia de marcas se realice de forma sutil, discreta o de fondo, con el objetivo de que la percepción por parte del receptor exista, pero no se aprecie invasiva. Sin embargo, empiezan a aparecer ejemplos en lo que se apuesta por una presencia evidente, agresiva, de frente, que obvia la presencia de la marca en la narración. Este avance, este grito de las marcas para recordar que están presentes, resulta relevante si tenemos en cuenta que, en otros soportes como el brand placement televisivo, la idea es que la presencia de la marca sea sutil.

El videoclip, por tanto, se convierte en un producto innovador en lo que a brand placement se refiere, en especial por esta apuesta por la publicidad evidente, sin tapujos y sin ningún tipo de escondite. Se constata que las marcas van ganando el terreno a la narración y se transforman en un sujeto más de la narración audiovisual. Esta circunstancia abre una línea que nos platea una nueva pregunta de investigación: si las marcas huyen de la publicidad convencional por la saturación, ¿no se estarán "saturando" los videoclips de marcas y, además, sin regulación ni autorregulación? Los resultados de esta investigación muestran que existen evidencias de una presencia masiva de marcas en los videoclips que consume un sector joven y en proceso de creación de su identidad, lo que implica una necesaria vigilancia de estas estrategias.

La presencia de las marcas en los videoclips persigue, a través del brand placement, que los creativos puedan construir cierta personalidad alrededor del producto que coincida con los valores de los artistas que prescriben las marcas. En las economías capitalistas modernas, tal y como hemos visto, el arte y el comercio conviven para crear unos contenidos musicales que sirven para vender cualquier producto de la industria de bienes y servicios.

La publicidad puede marcarse como objetivo incidir sobre las ventas de forma directa, o bien puede orientarse a provocar efectos no comportamentales, esto es, reacciones psicológicas que, aunque no se traduzcan en ventas de forma inmediata, creen una predisposición favorable del consumidor hacia el producto o marca anunciada, como es el caso de los ejemplos descritos en este trabajo con 
marcas como Adidas, Levi's o Beats by Dree, en las que el producto condiciona la narrativa audiovisual del videoclip.

En definitiva, este trabajo, ${ }^{3}$ que muestra de forma descriptiva los datos sobre prescriptores, tiempos y presencias de las marcas y tipos de emplazamientos, deja una ventana abierta para seguir trabajando en esta línea y abre nuevas líneas de investigación alrededor de los sectores que sustentan las marcas más activas en brand placement musical, las diferencias que puedan existir entre años o las posibles consideraciones de género que se desprendan de dicho emplazamiento.

Cande Sánchez Olmos (cande.sanchez@ ua.es) es Premio Extraordinario de Doctorado, doctora internacional por la Universidad de Alicante en Comunicación Audiovisual y licenciada en Periodismo por la UAB. Es profesora de Semiótica de la Comunicación de Masas en la Universidad de Alicante y de In-

Jesús Segarra Saavedra (jesus.segarra@ ua.es) es Premio Extraordinario de Doctorado, doctor en Comunicación y licenciado en Publicidad y Relaciones Públicas (Universidad de Alicante). Profesor contratado doctor acreditado por ANECA. Docente y profesor de Comunicación en UA y UNIR. Investigador

Tatiana Hidalgo Marí (tatiana.hidalgo@ ua.es) es doctora en Comunicación Audiovisual y Publicidad por la Universidad de Alicante, máster en Comunicación e Industrias Creativas (UA 2012) y licenciada en Publicidad y RR PP (UA 2007). Es editora técnica adjunta de Revista Mediterránea de Comunicación y miembro del grupo de investigación Industrias Culturales Hoy: Producción, dustrias Creativas en el máster Comincrea. Ha publicado varios artículos y capítulos de libros relacionados con la música y la publicidad. Desde 2017 dirige el proyecto de investigación emergente de la UA "Pop brands: la evolución del brand placement en los videoclips de la Billboard Hot 100 (2005-2016)".

en el grupo COMPUBES (UA). Editor técnico adjunto de Revista Mediterránea de Comunicación, coeditada por la UA y la UNIZAR, editor adjunto de Revista Health and Addictions (INID de la UMH), colaborador de Revista Latina de Comunicación Social (ULL) y socio de AE-IC, SLCS, PLATCOM, RELAIP y RIdHC.

Difusión, Gestión y Consumo de Productos Culturales en la Era de la Información (IICCXXI), de la Universidad de Alicante y del Observatorio de Ficción Española y Nuevas Tecnologías (OFENT) de la Universitat Autònoma de Barcelona. Sus principales líneas de investigación se centran en los estudios sobre la ficción televisiva con perspectiva de género y el análisis del discurso.

3 Proyecto emergente del Vicerrectorado de Investigación y Transferencia de Conocimiento de la Universidad de Alicante "Pop Brands: La evolución del brand placement en los videoclips del Billboard Hot 100 (2003-2016)". Referencia: GRE 16-24.

Especial agradecimiento a Patricia Palomares Sánchez, graduada en Publicidad y Relaciones Públicas por la UA y colaboradora de esta investigación. 


\section{Bibliografía}

Baek, T. H. y Morimoto, M. (2012). "Stay Away from Me". Journal of Advertising, 41 (1), pp. 59-76.

Dix, E. y Phau, I. (2010). "Television Advertising Avoidance: Advancing Research Methodology". Journal of Promotion Management, 16, pp. 114-133. Doi: <https://doi.org/1 0.1080/10496490903574013>.

Englis, B. G. (1991). "Music Television and Its Influences on Consumers, Consumer Culture, and the Transmission of Consumption Messages". Advances in Consumer Research, 18 (1), pp. 111-114.

Goodwin, A. (1993). Dancing in the Distraction Factory: Music Television and Popular Culture. Londres: Routledge.

Holden, J. (2007). Publicly-Funded Culture and the Creative Industries. Londres: Arts Council of England.

Hollenbeck, C. R. y Zinkhan, G. M. (2006). "Consumer Activism on the Internet: The Role of Anti-Brand Communities". Advances in Consumer Research, 33 (1), pp. 479-485.

Hudson, S. y Hudson, D. (2006). "Branded Entertainment: A New Advertising Technique or Product Placement in Disguise?". Journal of Marketing Management, 22 (5/6), pp 489-504.

IFPI (2016). Digital Music Report. Available in: <http://www.ifpi.org/downloads/DigitalMusic-Report-2014.pdf $>$. Consultado el 4 de abril de 2015.

Kaplan, A. (1987). Rocking Around the Clock: Music Television, Posmodernism and Consumer Culture. Londres: Methuen Inc.

Klein, N. (2000). No logo: el poder de las marcas. Barcelona: Paidós.

Krishen, A. S. y Sirgy, M. J. (2016). “Identifying with the Brand Placed in Music Videos Makes Me Like the Brand". Journal of Current Issues \& Research in Advertising, 37 (1), pp. 45-58. Doi: <10.1080/10641734.2015.1119768>.

Lehu, J. M. (2007). Branded Entertainment: Product Placement \& Branded Strategy in the En- tertainment Business. Londres: Kogan Page Publishers.

Madinabeitia, E. (2005). "Saturación y eficacia publicitaria". Telos: Cuadernos de Comunicación e Innovación, 64, pp. 9-11.

Marques, S. y Sedeño, A. (2017). Emplazamiento de producto y videoclip musical: Análisis de videos musicales más visualizados en YouTube (2011-2015). AdComunica, Revista Científica de Estrategias, Tendencias e Innovación en Comunicación, 14, pp. 97-117. Doi: <http:// dx.doi.org/10.6035/2174-0992.2017.14.6> .

Matthes, J. y Naderer, B. (2016). "Product Placement Disclosures: Exploring the Moderating Effect of Placement Frequency on Brand Responses Via Persuasion Knowledge". International Journal of Advertising, 35 (2), pp. 185199. Doi: <https://doi.org/10.1080/02650487. 2015.1071947>.

Moor, E. (2003). Branded Spaces the Scope of New Marketing. Journal of Consumer Culture, 3 (1), pp. 39-60. Doi: <https://doi.org/10.1177 /1469540503003001929>.

MyTop100Videos (2018). Most Viewed Videos of All Time. Disponible en <https://goo. gl/VT5NrA>. Consultado el 19 de noviembre de 2018.

Pérez, J. P.; Navarrete, J. L. y Gómez, F. J. (2014). "El 'product placement' en el videoclip: análisis de contenido del emplazamiento de marca en los vídeos musicales preferidos por los usuarios de YouTube". Doxa. comunicación, 18, pp. 83-104.

Pino, C. del y Olivares, F. (2006). 'Brand placement': integración de marcas en la ficción audiovisual. Evolución, casos, estrategias y tendencias. Barcelona: Gedisa.

Pino, C. del; Castelló, A. y Ramos, I. (2013). La comunicación en cambio constante. Madrid: Fragua.

PQ Media (2018, noviembre 22). PQ Media Executive Summary Download (comunicación personal). 
Regueira, J. (2012). El contenido como herramienta eficaz de comunicación de marca. Análisis teórico y empírico. Disponible en <https://goo. gl/JT389k>. Consultado el 16 de agosto de 2018.

Sánchez Olmos, C. (2016). "Videoclip publicitario, 'pop star' de la publicidad". Ctrl: Control \& Strategias, 636, pp. 82-85.

-. (2018). "Formatos y sectores en el 'branded content' musical: Videoclip y moda, pop stars de la musicidad". Revista Mediterránea de Comunicación/Mediterranean Journal of Communication. 9 (1), pp. 305-319. Doi: <https://www.doi.org/10.14198/MEDCOM2018.9.2.5>.

Sierra y Bravo (2005). Técnicas de investigación social: teoría y ejercicios. Madrid: Paraninfo.

Smit, E.; Reijmersdal. E. y Neijens, P. (2009). "Today's Practice of Brand Placement and the Industry Behind It". International Journal of Advertising, 28:5, pp. 761-782. DOI: 10.2501/S0265048709200898.

Soba, M. y Aydin, M. (2013). "Product Placement Efficiency in Marketing Communica- tion Strategy". International Journal of Business

\& Management, 8 (12), pp. 111-116.

Solana, D. (2010). Postpublicidad: reflexiones sobre una nueva cultura publicitaria en la era digital. Barcelona: Daniel Solana.

Thornton, C. y Burkhalter, J. (2011). “Emotion and Identification in Advertising: Must Be the Music: The Impact of Brand-Artist Association and Product Prestige on Consumer Responses to Music Video Brand placement". American Marketing Association Summer Educators' Conference Proceedings, 22, pp. 36-37.

-. (2015). "Must Be the Music: Examining the Placement Effects of Character-Brand Association and Brand Prestige on Consumer Brand Interest Within the Music Video Context". Journal of Promotion Management, 21:1, pp. 126-141. Doi: 10.1080/10496491.2014.971212.

Vernallis, C. (2013). Unruly Media: YouTube, Music Video, and the New Digital Cinema. Nueva York: Oxford University Press.

Viñuela, E. (2013). "El videoclip del siglo XXI: el consumo musical de la televisión a internet". Musiker, 20, pp. 167-185. 
